\section{Neue Option bei ALL}

Die Prognose bei akuter lymphatischer Leukämie (ALL) hängt von dem Erreichen einer molekularen Remission mit der ersten Induktions- und Konsolidierungs-Chemotherapie ab. Dies gelingt jedoch nicht immer. Mit Blinatumomab konnte in einer Phase-II-Studie bei 16 von 20 Patienten mit B-Vorläuferzell-ALL in hämatologischer Remission, aber mit molekularem Versagen nach Standard-Induktion und Konsolidierung eine molekulare Remission erreicht werden. Nach $33 \mathrm{Mo}-$ naten betrug das rezidivfreie Überleben dieser Patienten 19 Monate, der übrigen nur 3 Monate [Topp $\mathrm{M}$ et al. Blood. 2012;120(26):5185-7].

Nach Informationen von Amgen

\section{Empfehlung erhalten}

Der Ausschuss für Humanmedizin (CHMP) der Europäischen Arzneimittel-Agentur (EMA) hat sich für die $\mathrm{Zu}$ lassung der subkutanen Formulierung von Trastuzumab beim HER2-positiven Mammakarzinom ausgesprochen. Die s.c.-Applikation soll eine schnellere und weniger schmerzhafte Therapie ermöglichen. Studiendaten zeigen, dass die subkutane Therapie mit der bewährten Wirksamkeit und Sicherheit des Antikörpers einhergeht [Ismael G et al. Lancet Oncol. 2012;13(9):869. 78]. Die Zulassung wird im Herbst 2013 erwartet.

Nach Informationen von Roche

\section{Lymphom-Studie}

Takeda hat im Mai 2013 eine nicht interventionelle Studie zur Therapie mit Brentuximab Vedotin (Adcetris ${ }^{\circledast}$ ) gestartet. Ziel der Studie ist es, wichtige Erkenntnisse zur Versorgung von $\mathrm{Pa}$ tienten mit Hodgkin Lymphom und systemischem anaplastischem großzelligem Lymphom unter alltäglichen Praxisbedingungen zu gewinnen. Die Rekrutierung der Patienten wird voraussichtlich im Mai 2014 abgeschlossen sein.

Nach Informationen von Takeda

Diagnostische Marker

\title{
Sichere Tests als Basis zielgerichteter Therapien gefragt
}

\section{Vollautomatisierung und Test- Kits erleichtern die Arbeit des Pathologen, aber es können trotzdem Probleme auftauchen. Die diagnostische Genauigkeit ist keineswegs bei allen Tests und bei allen Laboren gleich.}

Neben der qualitätsgesicherten Mammakarzinomdiagnostik durch diagnostische Tests in der Immunhistologie (HER2) und der Molekularpathologie (HER2-FISH/ $\mathrm{CISH} / \mathrm{SISH})$, beruhen heute weitere Krebstherapien auf Biomarker-Testungen, wie z. B. BRAF-Mutationsanalyse, KRASoder EGFR-Mutationstest.

So entscheidet das Ergebnis der BRAFMutationsanalyse nicht nur über die Therapie, sondern auch über die Lebenserwartung der Patienten mit metastasiertem Melanom. Die Überlebenszeit von Patienten, die bei nachgewiesener BRAF-V600Mutation mit einem BRAF-Inhibitor wie z.B. Vemurafenib (Zelboraf ${ }^{\varpi}$ ) zielgerichtet behandelt werden, ist mit 16 Monaten doppelt so lange wie bei den mit der Standard-Chemotherapie Behandelten, erklärte Ralf Gutzmer, Hannover [Sosman JA et al. N Engl J Med. 2012;366(8):707-14]. Deshalb wurden BRAF-Testungen im Januar 2013 auch in die S3-Leitlinie „Diagnostik, Therapie und Nachsorge des Melanoms“ aufgenommen. BRAF-Mutationen treten besonders oft bei jüngeren Patienten auf. „Daher hinterfrage ich einen negativen BRAF-Befund gerade bei jüngeren Patienten besonders", sagt Gutzmer. Der Cobas-4800-BRAF-V600-Mutations-Test habe in Vergleichsuntersuchungen bereits einen mutierten Zellanteil von $5 \%$ erkannt, die Sanger-Sequenzierung erst einen Anteil von 20\% und die Pyrosequenzierung $10 \%$.

Ulrike Tietze

Symposium „HER2 - BRAF - EGFR: Bedeutung neuer Therapieansätze für die Qualitätssicherung in der diagnostischen Routine", Deutsche Pathologietage 2013, Berlin, 20.04.2013; Veranstalter: Roche

Myelodysplastisches Syndrom

\section{Chelat-Therapie bei Eisenüberladung}

\section{Eisenüberladung bei transfusions- abhängigen Patienten mit myelo- dysplastischem Syndrom (MDS) ist mit einer ungünstigen Prognose assoziiert. Eine adäquate Chelat- Therapie kann Hämatopoese und Überleben verbessern.}

Der Überlebenseffekt einer Chelat-Therapie sei in verschiedenen Studien eindeutig belegt worden, berichtete Valeria Santini, Florenz/Italien. Eine Matched-Pair-Analyse des Düsseldorfer MDS-Registers weise darauf hin, dass mit Chelator behandelte MDS-Patienten im Median 26 Monate länger leben als ohne diese Therapie (75 vs. 49 Monate; $p=0,002$ ) [Neukirchen J et al. Leuk Res. 2012;36(8);1067-70]. „Eine Eisenüberladung scheint ein unabhängiger prognostischer Faktor für das Gesamtüberleben zu sein“, so die Expertin. Bei Se-
rumferritin-Werten $<300 \mathrm{ng} / \mathrm{ml}$ ist der Benefit einer Chelat-Therapie besonders ausgeprägt [de Swart L et al. Blood. (ASH Annual Meeting Abstracts) 2011; 118(21): \#2775]. Mit dem nur einmal täglich oral einzunehmenden Eisenchelator Deferasirox $\left(\right.$ Exjade $\left.^{\star}\right)$ sei die Chelattherapie einfacher geworden, betonte Daniela Cilloni, Turin/Italien. Um eine hohe Adhärenz zu erreichen, sollten die Patienten darauf hingewiesen werden, dass zu Therapiebeginn Übelkeit, Erbrechen oder Diarrhö auftreten können, sich aber unter fortdauernder Behandlung meistens zurückbilden.

Abdol A. Ameri

Plenarsitzung IV: „Treatment of low risk MDS patients - the standard, the new", $12^{\text {th }}$ International Symposium on Myelodysplastic Syndromes der MDS-Foundation, Berlin, 10.05.2013; Veranstalter: Novartis Pharma 